\title{
Implementation of WAP Technology in Hospital Premises: A Proposed Approach
}

\author{
Shivali Dhaka \\ Assistant Professor \\ Amity University, Noida \\ India
}

\author{
Ashima Gambhir \\ Assistant Professor \\ Amity University, Noida \\ India
}

\begin{abstract}
The wireless application protocol (WAP) is now the leading standard for information services and provides a better environment to significantly improve the wireless data market. In this paper, the author proposed and analyzed a novel approach for sharing \& distributing information between patients and doctors in order to reduce the inconvenience and enhance quality of information in hospitals by using wireless application protocol (WAP) technology. This paper describes a WAP network for distributing information to ensure that patients and doctors can access information at any time and any location on adhoc basis; they only need a mobile phone. Patients mostly agreed WAP service is possible to utilize some of the Hospitals problems in providing useful information such as timings of doctors, booking an appointment, alerts of Lab reports, notification of medications, announcement, news and about different specialists. Also getting notification about their appointments \& surgeries will benefit the doctors. Therefore, Hospital campus can provide and offer information for mobile users as value added services.
\end{abstract}

\section{Keywords}

WAP, Wireless Markup Language, WAE, WSP, WTP, WDP.

\section{INTRODUCTION}

\begin{abstract}
The Wireless Application Protocol (WAP) [1] is the open-source international standard communication environment for wireless devices. WAP was published by the WAP Forum, founded in 1997 by Ericsson, Motorola, Nokia, and Unwired Planet and is a result of continuous work by the WAP Forum to define an industry-wide specification for developing applications that operate over wireless communication networks. WAP defines a set of protocols in transport, session and application layers to meet the challenges in advanced services, differentiation and fast/flexible service creation. It is an application environment and a set of communication protocols for wireless devices designed to give manufacturer, vendor, and technology-independent access to the Internet and advanced telephony services. WAP is typically accessed via mobile phones or Internet-connected Personal Digital Assistants (PDAs). WAP web browsers read Wireless Markup Language (WML) web documents, which are specially programmed for use with mobile cellular devices. There are six parts that combine to make the WAP protocol suite: Wireless Application Environment (WAE), Wireless Session Protocol (WSP), Wireless Transaction Protocol (WTP), Wireless Transport Layer Security (WTLS), Wireless Datagram Protocol (WDP) and the wireless network [2] itself as shown in figure 1. WAP can be implemented on any operating system.
\end{abstract}

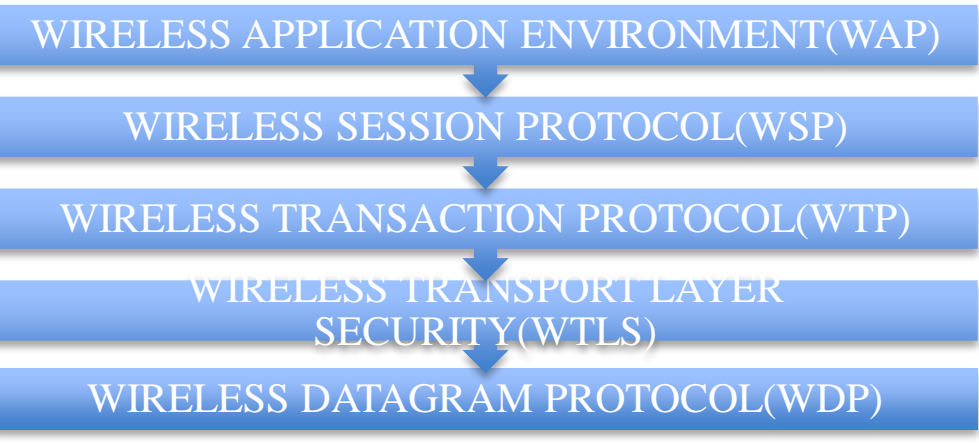

Fig 1: WAP protocol stack

This paper focuses on development of a mobile browser in Hospital Premises that supports WAP and web service. The technology will help patients to book their appointments, check timings of doctors and specialists, and check their reports and even complete their registration process on mobile device itself. It performs managing Patient's information system in the wireless environment. Patients and Doctors will get more value-added service, which are easy to use directly from a mobile phone to access information at any time, any locations. The current contentions are: i) information cannot be access at any time, at any locations i.e immobility; and ii) registration and results of reports need to be collected from hospital itself. This research is to improve the convenient for patient information retrieval, for which WAP has provided a promising solution. It saves the time of people and makes them feel convenient to access online information via mobile device.

This paper explains the approach of WAP technology for making information easily available to all patients as well as doctors in Hospitals. It involves WAP interface and design that makes use of WAP gateway to pass information between server and mobile device.

The work is organized as: In section 2, the author discussed the Related Works with respective to this WAP technology and analyzed WAP techniques, it's used in mobile commerce and its applications. In section 3, the author follows methodology 
procedure to explain framework of WAP services used in hospital network via WAP gateway. Finally, section 4 concludes the paper.

\section{RELATED WORKS}

WAP used in wireless communication networks uses WTLS, a wireless variant of SSL/TLS protocol to secure the communication between mobile phone and other parts of web architecture. The WAP Gateway decrypts all the WTLS traffic and encrypts all the SSL/TLS traffic. Both WAP Gateways and servers have to be upgraded kindly. Both the user and web server on the Internet trust the WAP Gateway for secure communication [3]. Wireless or mobile e-commerce is concerned with conducting transactions and other business activities with WAP technology for which we required a truly global wireless communication infrastructure with sufficiently high bandwidth to satisfy the needs of wireless and m-commerce applications [4]. For purchasing and vending of goods and services through mobile apparatus in new commerce pattern, an application called mobile commerce is invented which covers any kind of business transaction or information exchange using wireless and mobile technologies [5]. WAP based mobile GIS can be described as mobile users can perform the almost same functionality as of web GIS but in a mobile environment at any time, any place and without limitation of operating system and wired link [6]. Existed Computing Systems such as databases; legacy application and web services are required to be assimilated for advance mobile application. Life cycle of mobile application moves much faster. Developers stay in touch with latest developments, news and trends in their area of work with help of this. Even the security and application testing are more stimulating and interesting in mobile application than in web applications. To meet requirement of software development companies a new method called Agile methodologies were introduced [7]. Wireless Internet news sites delivered using the wireless application protocol by making the use of WebQual $/ \mathrm{m}$ instrument. It is used to assess the customer perception of WAP site and user oriented qualities that are assessed via an online questionnaire. Enhancements to service technologies like WAP will allow interactive and multimedia-rich applications for mobile phones [8]. M-Learning can be enhanced by raising the usability of mobile terminals as poor usability disturbs the learning process because the energy of uses directed to use of system instead of learning [9]. A mobile Internet application called MAP based on WAP technology provides flexible and scalable environment for mobile Internet applications to operate in. It makes possible to push critical information such as news and stock prices to mobile users in real time [10].

\section{FRAMEWORK AND ARCHITECTURE}

The research work proposed by this paper is for WAP technology for implementing Patients information system in hospital premises. The WAP services include four components in development phase i) Internet server ii) WAP Gateway iii) WAP server and iv) GSM Network i.e. link to existing to hospital network. In traditional web service the users request information from the web server directly from PC with the use of Internet or intranet. But with the emergence of WAP technology, the users are retrieving information in their mobile device at any time and any location. The network architecture design of the hospital premise WAP based Patients Information System can be understood by two main structures i) Framework of WAP Technology as Information Service ii) WAP architecture for hospital premise. WAP gateway utilizes web proxy technology to provide efficient wireless access to the Internet. WAP gateway [11] serves as a proxy to handle the requests from the WAP handset and passes the requests to the origin server. On the Internet side, the WAP gateway translates requests from the WAP protocol stack to the Internet protocol stack. On the wireless network site, the encoder/decoder in the WAP gateway performs WML text and bytecode conversion to reduce the information transmitted over the wireless network. The WAP gateway typically supports the DNS service to resolve the domain names used in URLs. It also provides quick response to the WAP handsets by aggregating data from different origin servers, and by caching frequently used information. Figure 2 shows the framework of information services provided by WAP technology in which the information is distributed between patients and doctors via WAP gateway using hospital network i.e GSM/3G which makes possible for mobile users to avail the service.

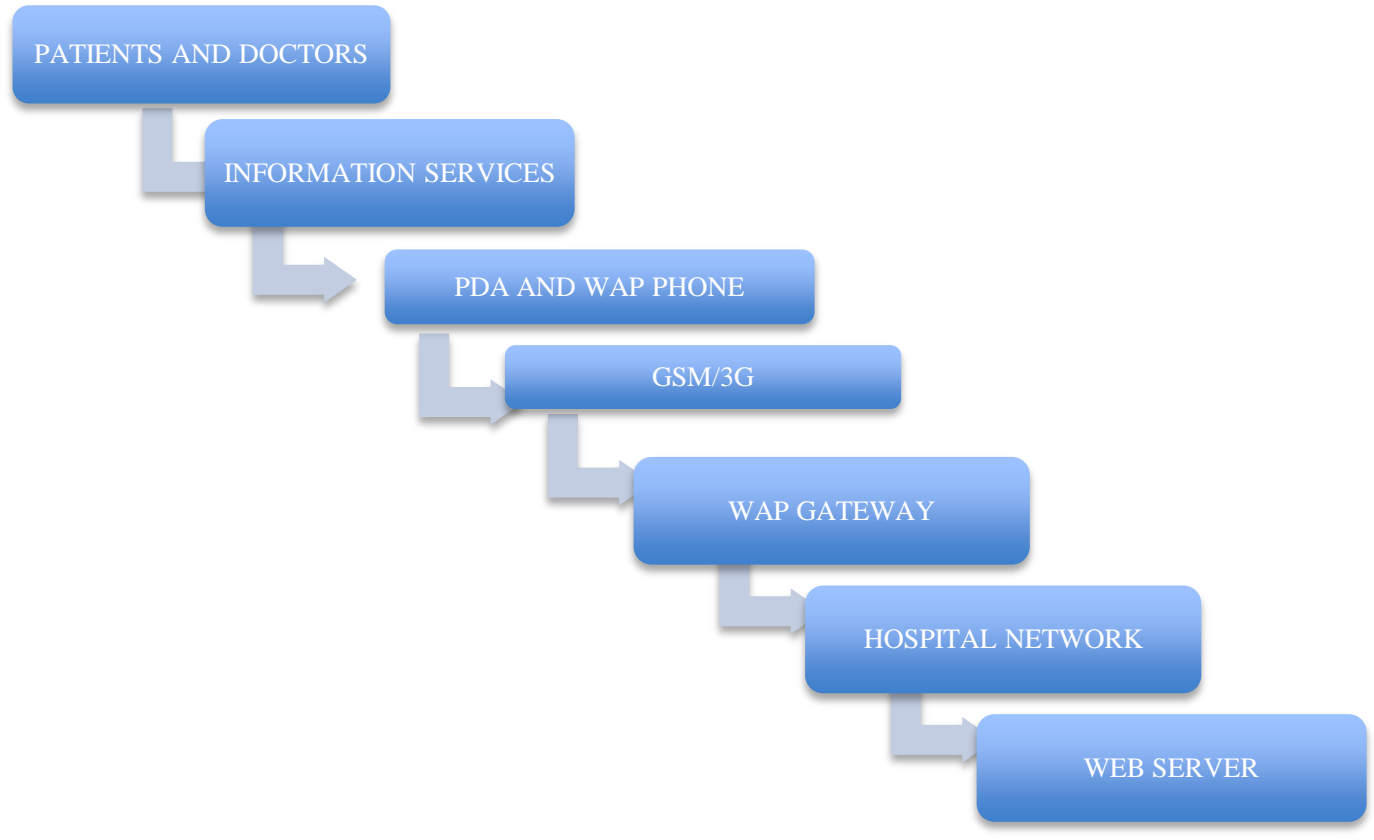

Fig 2: Framework of WAP Technology as Information Service 
Figure 3 shows the WAP service environment to retrieve information at hospital based on mobility approaches. Hospital network is accessed by all the patients and doctors via WAP gateway that is acting as the main agent for encoding the request and decoding the reply.

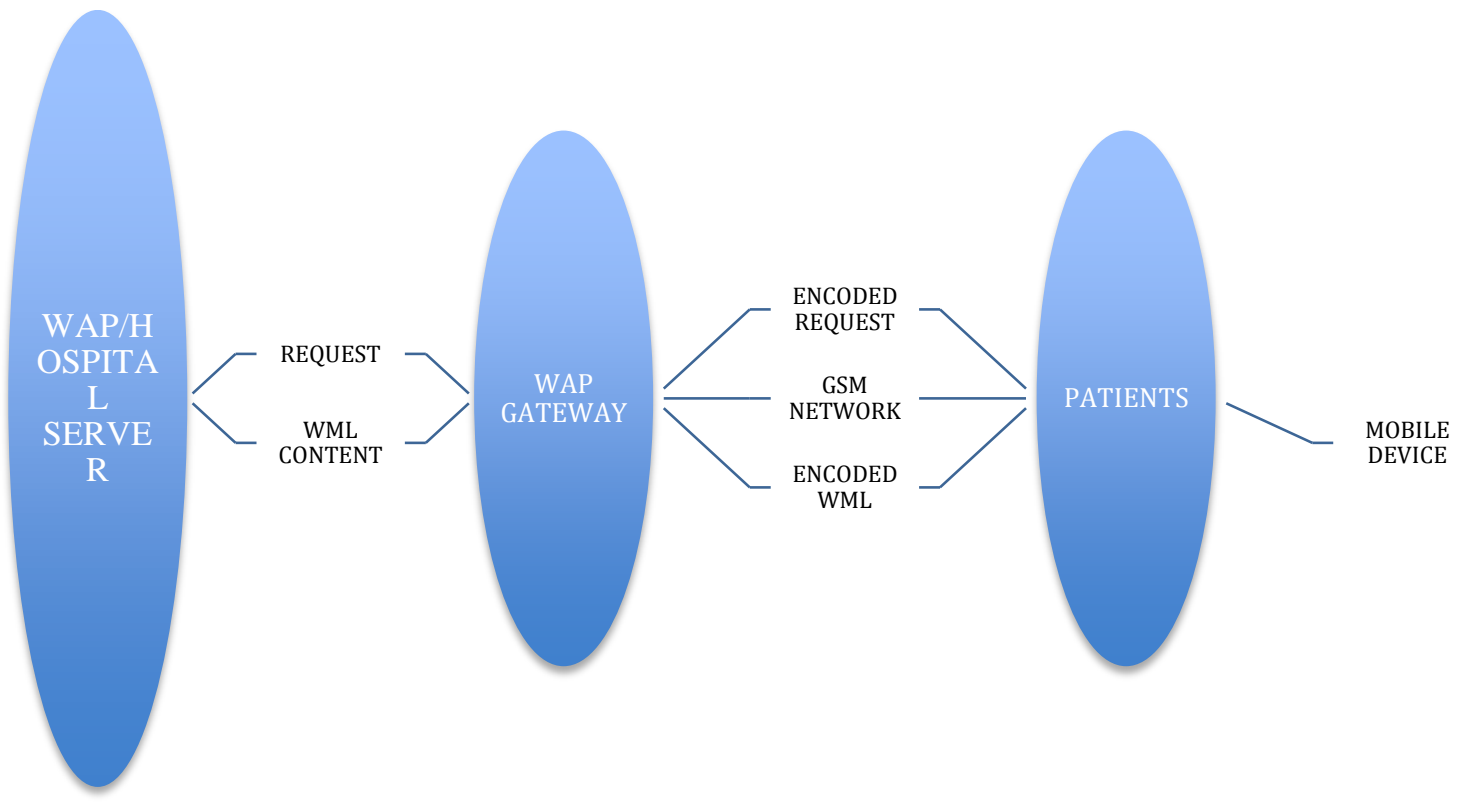

Fig 3: WAP architecture for hospital premise

The main objective of this WAP technique is i) to provide an easier method to access information ii) provide a WAP based information retrieval system to the patients and doctors at any location iii) provide easy key access and menu driven interface as it will apply in the system to minimize text entry by keypad.

\section{CONCLUSION}

In this paper the main focus in on the development of mobile browser in hospital premises that suppose WAP as well as web service. The author presented WAP based Patients Information System, a mobile web browser for handheld devices with no mobility problem so that patients are able to retrieve information at any time. The practical value of mobility in hospitals will be greater in future because mobile terminals are flexible to use and they enable real time and place independence. This information system enabled mobile patients to avail much valuable information and a large number of services over mobile networks. This WAP application provides patients to do their registrations, to book their appointments, to receive notifications of OPD timings of concerned doctors, alerts of lab records and notification of prescribed medication. It can use to retrieving information based on ad-hoc and mobility environment. It provides a user-friendly graphical and text interface, which is easy to use and presents a comprehensive list of constraints structured for analyzing WAP services architecture. The system can be further enhanced to become more efficient and widely used system. Many improvement and enhancement can be made to the existing WAP system to meet the changing needs of patients.

\section{REFERENCES}

[1] WAP Service Indication, Version 08-Nov-1999, Wireless Application Protocol Forum, Ltd., 1999.

[2] WAP Push Architectural Overview, Version 08-Nov-1999, Wireless Application Protocol Forum, Ltd., 1999.

[3] Dave Singelee and Bart Preneel[2005],"The Wireless Application Protocol", International Journal of Network Security, Vol.1,No.3,PP.161-165.
[4] Peter Tarasewich, Robert C. Nickerson, Merrill Warkentin [2001],"Wireless/Mobile E-Commerce: Technologies, Applications and Issues",Seventh Americas Conference on information systems.

[5] Muhammad Salam, Nasir Rashid, Fakhre Alam, M.raees Khan [2013], “ Dynamic WAP Page generation: A Proposed Solution For Small Mobile Screen and Usability Issues", International Journal of Computer Applications(0975-8887), Volume 73-No. 11.

[6] Wang Fangxiong, Bian Fuling and Hou Yingzi, "A Distributed Architecture for WAP based Mobile GIS", Commission VI, WG VI/4.

[7] Harleen K. Flora, Dr. Swati V. Chande [2013],"A Review and analysis on mobile application development process using agile methodologies", International journal of research in computer science, eISSN 2249-8265 Volume 3 Issue 2 pp.918.

[8] Stuart J.Barnes, Kenny Liu, Richard T. Vidgen [2001], "Evaluationg Wap News Sites: The Webqual/M Approach", Global Co-Operation in the New Millennium ,The $9^{\text {th }}$ European Conference on Information Systems, June 27-29.

[9] Kristian K. 2002. "Evaluating WAP Usability: "What Usability?" IEEE International Workshop on Wireless and Mobile Technologies in Education (WMTE'02), pp. 169.

[10] Jarvenpaa, S.L., K.R. Lang, Y. Takeda \& V. Tuunainen [2003], "Mobile Commerce at Crossroads", Communications of the ACM. Vol. 26, no. 12, pp. 41- 44.

[11] WAP Architecture, Version 30-Apr-1998, Wireless Application Protocol Forum, Ltd., 1998. 